\title{
New Signal Processing Techniques for Improved Precision of Noninvasive Impedance Cardiography
}

\author{
J.H. Nagel, L.Y. Shyu, S.P. Reddy, B.E. Hurwitz \\ P.M. McCabe, and N. Schneiderman \\ Behavioral Medicine Research Center \\ Departments of Biomedical Engineering, Psychology and Medicine \\ University of Miami \\ Coral Gables, Florida
}

(Received 2/2/89; Revised 3/29/89)

\begin{abstract}
Impedance cardiographic determination of clinically important cardiac parameters such as systolic time intervals, stroke volume, and related cardiovascular parameters has not yet found adequate application in clinical practice, since its theoretical basis remains controversial, and the precision of beat-to-beat parameter estimation has until recently suffered under severe shortcomings of available signal processing techniques. High levels of noise and motion artifacts deteriorate signal quality and result in poor event detection. To improve the precision of impedance cardiography, new techniques for event detection and parameter estimation have been developed. Specifically, matched filtering and various signal segmentation and decomposition techniques have been tested on impedance signals with various levels of artificially superimposed noise and on actual recordings from subjects in a laboratory study of cardiovascular response to a cognitive challenge. Substantial improvement in the precision of impedance cardiography was obtained using the newly developed signal processing techniques. In addition, some preliminary evidence from comparisons of the impedance cardiogram with invasive aortic electromagnetic flow measurement in anesthetized rabbits is presented to address questions relating to the origin of the impedance signal.
\end{abstract}

Keywords-Impedance cardiography, Pattern recognition, Signal processing, Matched filter, Cardiac output.

\section{INTRODUCTION}

Impedance cardiography was introduced over 20 years ago as a noninvasive and unobtrusive technique for measuring systolic time intervals, cardiac output, and several related cardiovascular parameters, including contractility and peripheral resistance. In spite of intensive research, development, and experimental efforts, impedance cardiography has not yet found adequate application in clinical practice.

This research was supported by program project research grant HL36588, by research grant HL41335, and by research training grant HL07426 from the National Heart, Lung and Blood Institute of NIH.

Address correspondence to Joachim Nagel, Ph.D., Department of Biomedical Engineering, University of Miami, PO Box 248294, Coral Gables, FL 33124. 
Since its theoretical basis remains controversial, acceptance of the technique has relied on empirical validation. In general, comparisons of impedance cardiography with well-established invasive techniques have shown high correlations when averaged across reasonably healthy subjects $(20)$, although there has been some tendency to overestimate stroke volume $(2,5,7,12,17)$. The correlations have been much poorer in some abnormal patient populations $(6,8,15)$, and when applied in individual subjects $(1,3)$. Thus, the practical value of impedance cardiography for critical care monitoring and diagnostic purposes in individual patients has not yet been realized.

The two major potential sources of error in impedance cardiography are the theoretical model and the signal processing techniques that have been used to detect the events in the impedance signal. Our investigations have revealed serious shortcomings in the commonly used signal processing techniques, that lead to large errors in the determination of cardiac time intervals and stroke volume. It is our contention that the quality of the theoretic model on which impedance cardiographic flow measurement is based cannot be evaluated as long as its performance is obscured or marred by inadequate signal processing techniques. Thus, the first step in improving impedance cardiography is the development of precise signal processing techniques. The primary purpose of this paper is to describe improved signal processing techniques, thus providing the tools for further investigations of models of impedance cardiography. Since some of our results presented later suggest that changes in the model may also be necessary, initial investigations of the validity of the existing models for the origin of the impedance signal have been done by invasive animal experiments and are presented in this paper. The results reveal that some of the assumptions on which current models are based need to be adjusted for further improvement of the precision of impedance cardiography.

\section{THEORY AND METHODS}

Impedance cardiography is based upon characteristic changes of thoracic electric impedance $(Z)$, that occur as a function of cardiac activity. As can be seen from Fig. 1, distinctive landmarks in the impedance cardiogram (ICG), representing the first time derivative $d Z / d t$ of the thoracic impedance, enable the determination of cardiac time intervals. Electrocardiogram (ECG) and phonocardiogram (PCG) are shown as reference. Assuming that only left ventricular blood flow contributes to the observed impedance changes (10), stroke volume can be calculated by analyzing the impedance signal. The first widely accepted and still used formula for determining stroke volume has been developed in 1966 by Kubicek, Karnegis, Patterson, Witsoe, and Mattson (9):

$$
S V=\rho_{b}\left(L / Z_{0}\right)^{2} \operatorname{LVET}(d Z / d t)_{\max }
$$

where $S V$ is the stroke volume $(\mathrm{ml}), \rho_{b}$ is the resistivity of blood (ohm $\left.\mathrm{cm}\right), L$ is the distance between the recording electrodes $(\mathrm{cm}), Z_{0}$ is the baseline impedance between the recording electrodes (ohm), LVET is left ventricular ejection time (s), and $(d Z / d t)_{\max }$ is the absolute value of the maximum rate of change (slope) in the impedance signal for a given beat (ohm/s).

Referring to signal processing, the critical parameters in Kubicek's equation are $Z_{0}$, LVET, and $(d Z / d t)_{\max }$. Simple error calculation shows that the determination 


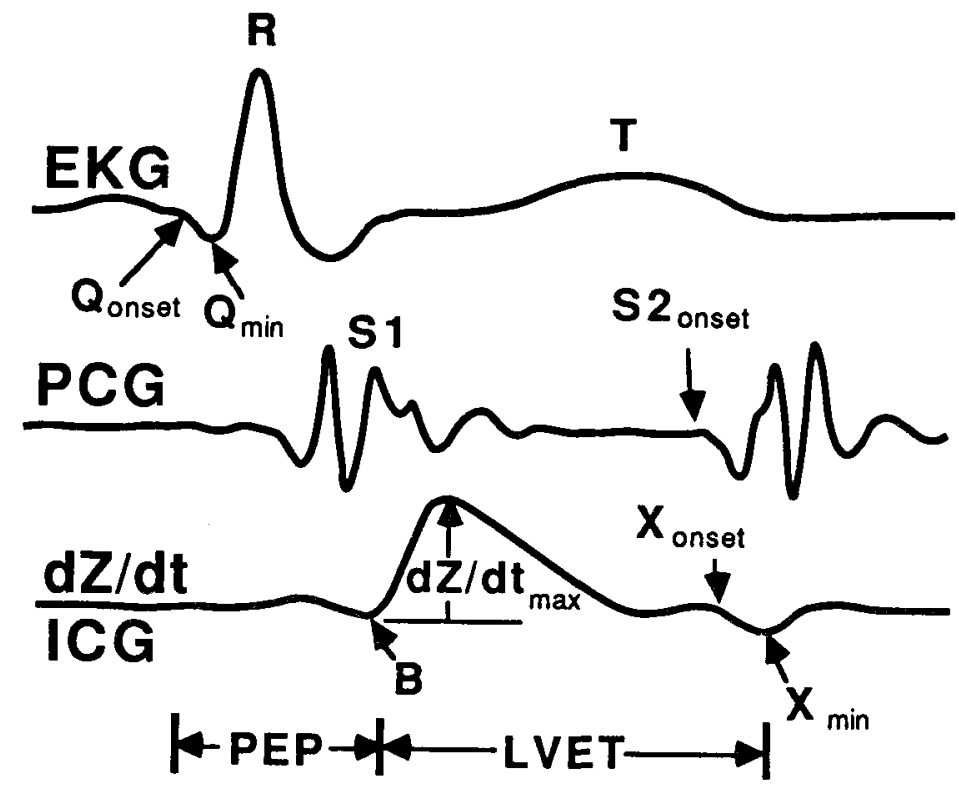

FIGURE 1. Drawing of electrocardiogram (ECG), phonocardiogram (PCG), and impedance cardiogram as typically recorded during electromechanical systole. Events depicted are $Q$ wave onset and minimum, $T$ wave $(T)$, first $(S 1)$ and second $(S 2)$ heart sounds, amplitude of the impedance cardiogram $\left(d Z / d t_{\text {max }}\right)$ measured from the value of the $B$ point, $X$ wave onset and minimum, and the systolic intervals pre-ejection period (PEP) and left ventricular ejection time (LVET).

of stroke volume is very sensitive to even small errors in these parameters. Assuming that baseline impedance, ventricular ejection time, and impedance slope are determined with a precision of $5 \%$, the worst case error adds up to approximately $20 \%$ of stroke volume. It will be shown later that conventional signal processing techniques result in even larger random and systematic errors, leaving only the hope that the worst case does not apply and the errors might cancel out. Making impedance cardiography a reliable diagnostic tool, however, requires improvement in the precision of signal processing techniques.

Two major problems exist in the determination of the required cardiac parameters from the ICG: (a) due to the unavoidable presence of noise and inherent variance of signal shape, demonstrated by characteristic examples in Fig. 2, localization of cardiac events, such as the opening and closing of the aortic valve, and determination of signal amplitude become susceptible to error; (b) respiration causes signal modulation as shown in Fig. 3, that needs to be removed before baseline impedance and slope can be determined with adequate precision. The influence of respiration on the impedance signal, however, additive superposition or multiplicative distortion, is unknown.

Previously used simple signal processing techniques such as threshold detection and search for maxima or minima have been inadequate to solve the problems as they introduce large amounts of jitter in localization of events and amplitude determination (4) and do not provide the means to remove respiratory modulation. Averaging techniques have been commonly used to overcome these problems of signal process- 

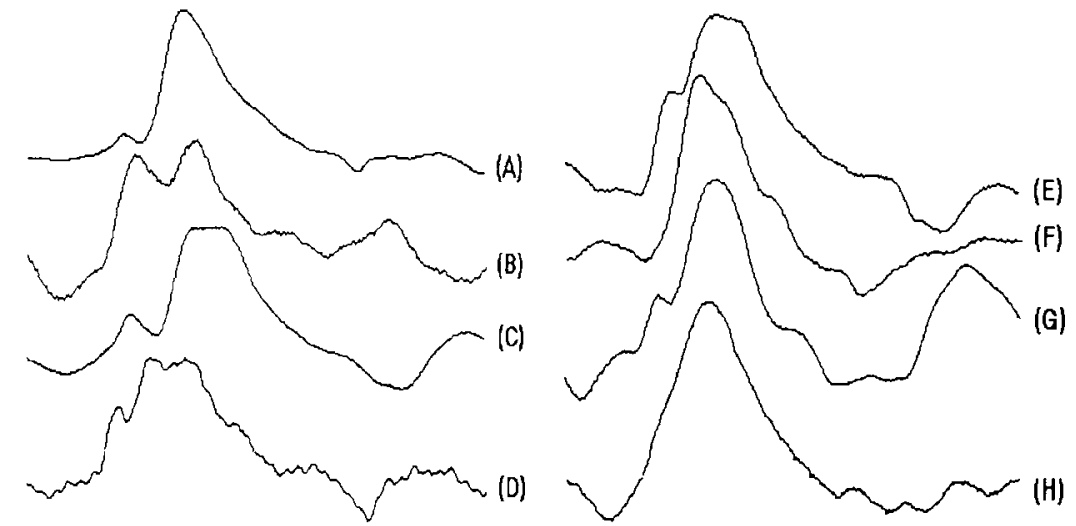

FIGURE 2. Physiological variations of actual $d Z / d t$ signals. (A) typical, (B) double peak, (C) flat peak, (D) noisy peak, (E) B point moving towards peak, (F) no $B$ point, (G) flattened $X$ wave (H) unclear position of $X$ wave.

ing $(18,22)$. However, inherent in the use of averaging is the expense of information about beat-to-beat variability and transient responses. Two different averaging techniques have been used: either beat-to-beat determined parameters have been averaged, or parameters have been extracted from ensemble averaged signals. The drawback of parameter averaging is that random errors disappear, but convergence to the correct values cannot be guaranteed since it does not prevent systematic errors of signal processing from influencing the single cardiac parameters. Alternatively, ensemble averaging of the ICG suffers from the difficulties associated with variability in signal shape and event latencies that can cause less distinctive events in the signal to disappear in the averaged signal. Some signal processing problems could be solved without the need of averaging by the development of more sophisticated techniques employing matched filter detection and various signal segmentation and decomposition techniques.

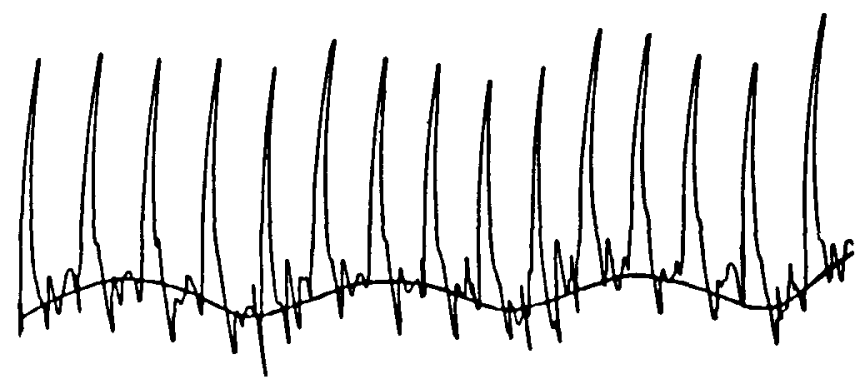

FIGURE 3. Respiratory modulation of impedance cardiogram. 


\section{Matched Filter Event Detection}

Improving the quality of signal processing techniques for event detection involves consideration of two problems: reliability and precision. Biological signals are always contaminated by noise and show inherent variations in shape. The recognition of distinctive events such as the ventricular electric systole, marked by the $R$ wave of an ECG, or the ventricular ejection phase, marked by the distinctive upstroke of the impedance cardiogram, can usually be done with high reliability and precision. Exact localization of the less distinctive events or wave onsets ( $Q$ wave, $B$ point, $X$ wave) and amplitude determination $\left(d Z / d t_{\max }\right)$, can represent a serious problem. As the characteristic features of these events are often obscured by noise, or change physiologically, not only precision but even reliability of detection deteriorates. In such cases reliability has often been maintained at the expense of precision, replacing the detection of the actual event by the detection of a related, neighboring event with distinctive features (e.g., $Q$ wave onset replaced by $R$ wave upstroke, or B point represented by zero-crossing of the impedance signal). Precision, of course, suffers by this substitution because systematic errors are introduced into the measurement. Figure 2 demonstrates the influence of noise and artifacts on the different landmarks in the ICG, which may change appearance sometimes even during a measurement. Reliable and precise event detection obviously requires techniques that offer improvement in signal-to-noise ratio (SNR), are insensitive to insignificant changes of signal shape, and adapt to major changes. All these features are offered by adaptive matched filters which can be realized by means of digital correlation with constant updating of the signal template.

If $x(t)$ and $y(t)$ represent two time domain signals, and $X(f)$ and $Y(f)$ are the corresponding frequency domain representations, then the cross-correlation function $R_{x y}(t)$ of the two signals is given by:

$$
R_{x y}(t)=F F T^{-1}\left[X(f) Y(f)^{*}\right]
$$

where ${ }^{*}$ specifies the complex conjugate function, and $F F T^{-1}$ refers to the inverse Fast Fourier Transform operation. If $y(t)$ represents the template $p(t)$ of an event that should be detected in $x(t)$ representing the measured signal consisting of a superposition $x(t)=s(t)+n(t)$ of the physiological signal $s(t)$ and noise or artifacts $n(t)$, then Eq. 2 becomes:

$$
R_{x p}(t)=F F T^{-1}\left[(S(f)+N(f)) P(f)^{*}\right]
$$

or, due to the linearity of the Fourier transform:

$$
R_{x p}(t)=F F T^{-1}\left[S(f) P(f)^{*}\right]+F F T^{-1}\left[N(f) P(f)^{*}\right] .
$$

Assuming that template and noise are uncorrelated, the second term on the right side of the equation disappears, and the noise does not influence the cross-correlation function:

$$
R_{x p}(t)=R_{s p}(t)=F F T^{-1}\left[S(f) P(f)^{*}\right]
$$




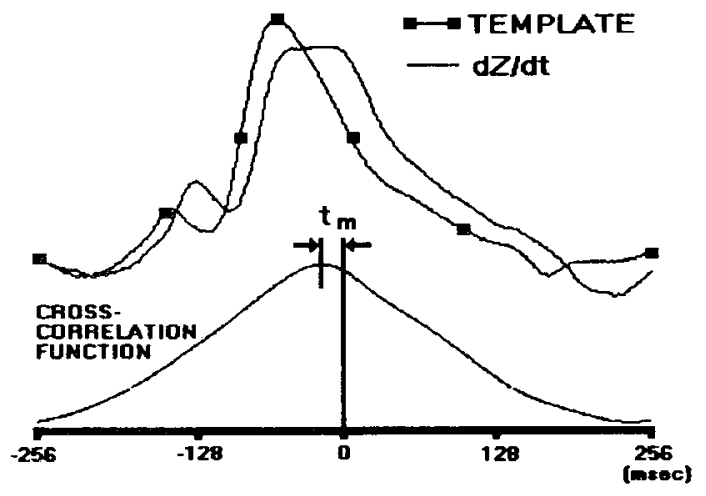

FIGURE 4. Matched filter event detection. The position of the maximum in the crosscorrelation function indicates the time delay between template and signal.

The time $t_{m}$ for which $R_{s p}\left(t_{m}\right)$ is the maximum value of the correlation function indicates the relative time shift between template and signal for which the highest similarity or best match is obtained (see Fig. 4). Thus, the exact location of the event can be determined. Since, ideally, any influence of noise is eliminated in the cross-correlation function, normalizing $R_{s p}(t)$ to one for the case of identical signals results in a quantitative measure of the similarity between template and signal, and specifies the reliability of the decision.

Application of matched filtering first requires the generation of a signal template. Two possibilities exist: (a) use of a standard signal and (b) generation of an individual template by coherent averaging. Detection based on a standard signal is not appropriate if the individual subject's signal does not correspond to the standard. Conversely, coherent averaging can only be performed, if a detection scheme is already available. This dilemma, where the development of a reliable detection procedure requires the detection to be already performed, might appear insoluble. The problem is, however, not as serious as it appears. Coherent averaging is much more error tolerant than the actual precise localization of the events. Thus, a simple threshold detector can be applied for the generation of a first template. Once a reasonable template is available, matched filtering may be applied for further signal detection, and by continuous updating of the template, now based on the precise detection technique, it is not only improved, it also adapts to time dependent changes (14).

Once the matched filter algorithm has been established, all of the different signal events can be detected. It is now just a matter of choosing the template of the desired event. In the cases of $\mathrm{B}$ point and $\mathrm{X}$ wave detection, an extension of the technique for subpattern recognition is recommended. Just applying an appropriate window for template matching often leaves a considerable influence of the main impedance complex, when $\mathrm{B}$ point or $\mathrm{X}$ wave are too close to this main complex. This influence can be removed by a curve fitting technique. As illustrated in Fig. 5, a curve is fitted to the main impedance complex based on the sample points outside the area of the event that has to be detected. This curve, which represents the ICG without the target event, is then subtracted from the whole signal. This leaves the pure signal contribution of the desired event that is easily recognized and located by matched filtering. 


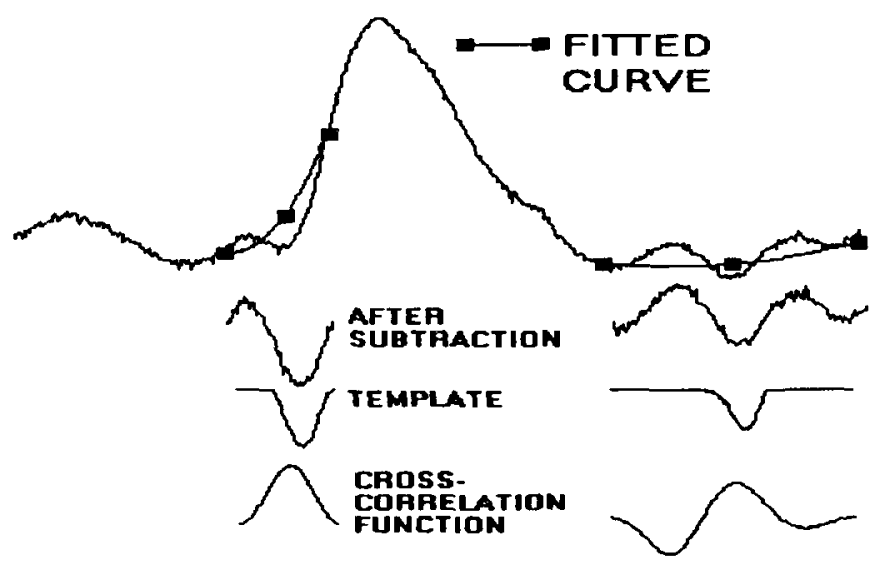

FIGURE 5. Segmentation of the impedance cardiogram by curve fitting for the matched filter detection of $B$ point and $X$ wave. A curve is fitted to the ICG disregarding the two events. After subtraction from the original signal, the events become clearly visible, and are detected through matched filtering.

\section{Correlative Amplitude Determination}

The determination of stroke volume from the impedance cardiogram requires the measurement of the maximum rate of change $\left(d Z / d t_{\max }\right)$ during the ejection period. As can be seen from Fig. 2, tracing D, noise and artifacts that deform the shape of the peak make it often difficult to determine the maxima. The problem of determining the amplitude of a noisy signal can also be solved by use of a correlation analysis (14). Assuming that an event or signal has been detected by matched filtering, thus eliminating the noise, then the output of the filter is given by Eq. 5 as

$$
R_{s p}(t)=F F T^{-1}\left[S(f) P(f)^{*}\right] .
$$

For the maximum at $t_{m}$, the value of the correlation function can be expressed in the time domain representation as:

$$
R_{s p}\left(t_{m}\right)=\int s(t) p\left(t_{m}+t\right) d t
$$

Assuming that template $p(t)$ and signal $s(t)$ are in phase, i.e., $t_{m}=0$, then

$$
R_{s p}(0)=\int s(t) p(t) d t
$$

Further, assuming that the signal has the same shape but different amplitude than the template, and any differences in appearance are due to noise that is eliminated by the matched filtering, i.e.

$$
s(t)=c p(t)
$$


then Eq. 7 becomes

$$
R_{s p}(0)=c \int p(t)^{2} d t
$$

or:

$$
R_{s p}(0)=c R_{p p}(0)
$$

The scale factor $c$ is determined by:

$$
c=R_{s p}(0) / R_{p p}(0)
$$

where $R_{p p}(0)$ represents the power of the template $p(t)$. This result means that dividing the maximum of the cross-correlation function by the power of the template results in the scale factor of the signal amplitude relative to the template. The advantage of this procedure over the direct measurement of the signal amplitude is that, ideally, any influence of noise or artifact is excluded. Since both template and crosscorrelation function are free of noise, the amplitude of the disturbed signal is determined by measuring the amplitude of the noise-free template and subsequent scaling:

$$
(d Z / d t)_{\max }=c(d Z / d t)_{\max , \text { template }}
$$

Due to the limited integration time for the cross-correlation function, the limited number of samples taken for the generation of templates, and the nonperfect match between template and actual signal, the real error in amplitude determination is not zero as it would be under ideal conditions. The actual error depends on the number of signal samples taken for template averaging and the signal statistics. But as Fig. 6

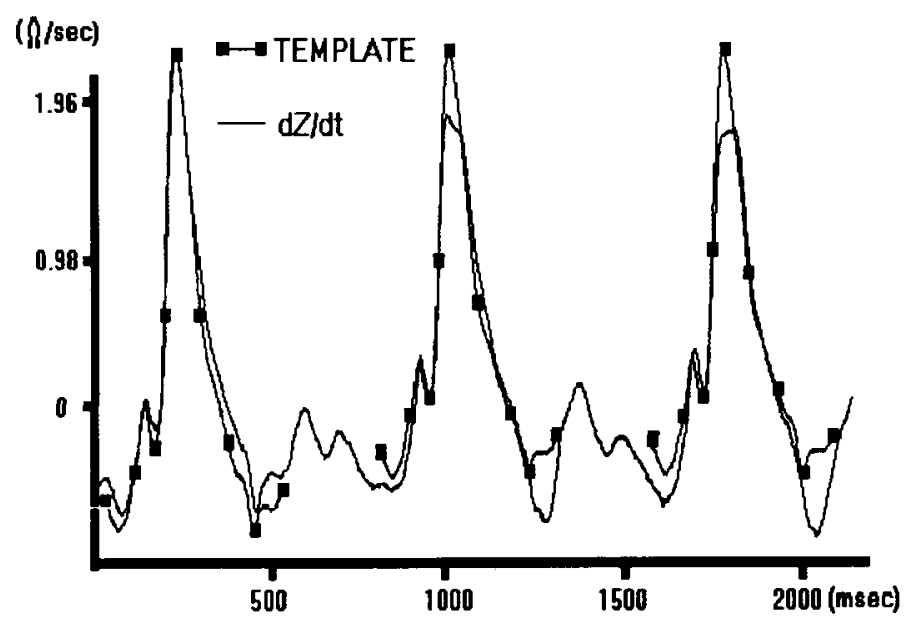

FIGURE 6. Amplitude determination of the ICG by correlative template matching. The distorted second and third peaks are restored by the template matching. 


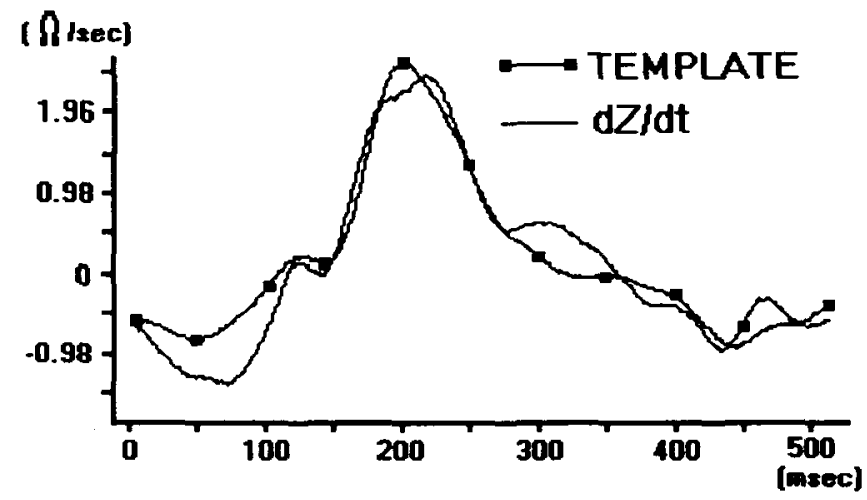

FIGURE 7. Cross correlation estimation of $d Z / d t_{\max }$. The template of the ICG is scaled such that its maximum represents the true maximum for the given distorted signal.

demonstrates, and the results presented later show, this procedure considerably improves determination of $d Z / d t_{\max }$. The impedance signal shown in Fig. 7 does not allow determination of $d Z / d t_{\max }$ with a high precision when conventional techniques are used. Even the exact location of the maximum is not readily apparent. Correlation analysis, however, clearly indicates the maximum and enables determination of its amplitude.

\section{Removal of Respiratory Modulation}

Under the influence of respiration, both baseline and amplitude of the impedance cardiogram show characteristic changes, leading to errors in the determination of $d Z / d t_{\max }$ and hence also in the stroke volume. So far, the only known technique for the elimination of respiratory modulation is coherent averaging of successive cycles. Besides the problems mentioned earlier, averaging leaves some basic problems that have not yet been examined in the published literature. Stroke volume calculated by measurement of the cardiac parameters of a single cardiac cycle varies depending on the phase of respiration during the measurement. Averaging many measurements equally spread over all respiratory phases eliminates the variations and results in a single value for stroke volume that has been assumed to be independent from respiration. This value, however, still remains influenced by the respiration pattern. Assuming, that the measurement during expiration reflects the correct stroke volume, then the stroke volume measured by averaging will be dependent on the relative length of inspiration time to expiration time. Hence, the averaged value will be systematically overestimated. Assuming a given baseline cardiac output, calculated by averaging over a certain period, and assuming further a changing pattern of respiration that may modulate $d Z / d t$ in a different way than in the first case, then we might calculate a changed cardiac output even if there is no change at all. Another shortcoming of averaging is that information about actual modulation of cardiac output as a function of respiration is lost. This would present a problem in a clinical test, for example, in which the use of paced respiration in diabetic patients is employed to determine the extent of autonomic degeneration. 


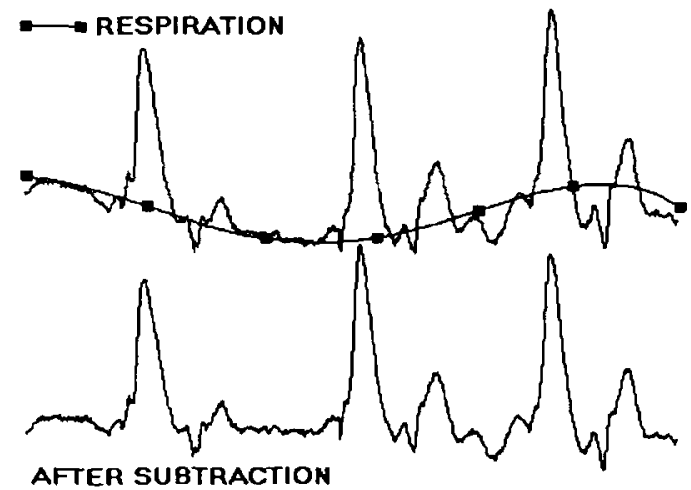

FIGURE 8. Removal of respiratory modulation from the impedance cardiogram. A curve is fitted to the baseline and subtracted from the ICG.

As demonstrated in Figs. 3 and 8, both baseline and amplitude of the ICG show a rhythmic modulation caused by respiration. Using a fixed $d Z / d t$ point within each cardiac cycle results in a curve that corresponds to respiration. Different curves are obtained, however, depending on whether the landmark in the $d Z / d t$ signal is selected on or close to the baseline, or whether the peak amplitude serves as the sampling point for the respiration curve. The mismatch reflects the differences in origin. The baseline shift of impedance is a consequence of changing thoracic geometry and current distribution, whereas the amplitude modulation of the peak may contain true respiratory dependent changes of stroke volume, which of course should not be eliminated by signal processing. As a consequence, it should be possible to reconstruct respiration from the baseline drift, and eliminate its disturbing influence on the ICG without losing the information about actual respiratory stroke volume modulation.

According to the sampling theorem, a signal can be completely reconstructed from its samples if the sampling frequency is at least twice the highest spectral component of the signal. Assuming an upper cut-off frequency for the respiration curve of 10 $\mathrm{Hz}$, sampling has to occur with at least $20 \mathrm{~Hz}$, or every $50 \mathrm{~ms}$. Though the above described correlation analysis of the ICG makes it possible to obtain fixed reference points, the required density of samples is not easily achieved, at least not during diastole, because the lack of distinctive events makes it difficult to separate noise and baseline signal. Since the actual purpose of reconstruction is to eliminate or at least reduce the respiratory influence on the determination of $d Z / d t_{\max }$, the procedure can be simplified by assuming a smaller bandwidth of the respiration signal and replacing exact reconstruction by approximation through curve fitting based on low sample density. As indicated in Fig. 8, spline fitting based on just the B point for each heartbeat leads to compensation of the baseline drift and consequently results in substantial improvement in amplitude determination. Since multiplicative distortions of the impedance cardiogram through respiration have not been reported in the literature, additive superposition was assumed in our investigation. Future work might delineate a possible multiplicative component and assess its impact on the results. Thus far, however, no signs of such distortion have been found. 


\section{PRECISION OF SIGNAL PROCESSING}

Validation of the new signal processing techniques was done in two steps. First, the improvement of the precision in event detection and amplitude determination was assessed by testing the new techniques against the simple detection algorithms that are commonly used and described in literature. Artificial signals were used in this study. Then the signal processing performance was tested in a laboratory study in which subjects were mentally challenged.

The artificial test signals used for the described evaluation of the new techniques were obtained by generating an ICG template from an actual impedance signal by ensemble averaging and subsequently superimposing noise and artifacts of known amplitude, thus simulating signals with different quality levels (see Fig. 9). The standard for the time and amplitude of the different events was obtained from the noisefree template. Event detection was then performed on the signals with different noise levels using both the simple and the new techniques, and the results were compared to the standard. Three different levels of signal distortion have been analyzed, specified by the corresponding signal-to-noise ratio (SNR), expressed as the ratio between signal amplitude and the standard deviation $\sigma$ of the noise signal. Table 1 and 2 show the results for the three levels of SNR: 5,10 , and 20 . The values in the tables have been obtained by averaging over 16 different signals.

As can be seen from Table 1, both mean error and mean absolute error for the detection of the $\mathrm{B}$ point in the impedance cardiogram exhibited negligible difference from the standard when the curve fitting technique was applied, whereas the simple technique of local minimum detection showed a substantial error, especially at higher levels of noise. The small difference between mean error and mean absolute error indicates that the simple technique introduces a systematic shift of the location of the $\mathrm{B}$ point toward the peak. This result is due to the fact that detection of the B point was programmed to begin searching midway down the $d Z / d t$ peak and continue

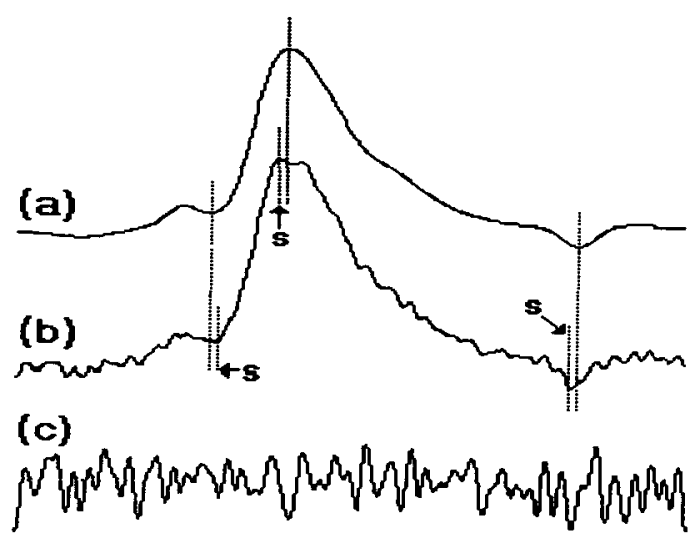

FIGURE 9. Generation of a $d Z / d t$ test signal. A noise function (c) of known amplitude is added to template (a) and results in test signal (b). The unlabeled markers represent $B$ point, maximum, and $X$ wave in the template, the markers labeled s represent the same events, as they are detected by the simple techniques. 
TABLE 1. Precision of simple and curve fitting methods: Effect of noise on the position of the B-point and the $X$ minimum of the $d Z / d t$.

\begin{tabular}{|c|c|c|c|c|c|}
\hline \multirow[b]{2}{*}{ Event } & \multirow[b]{2}{*}{ Method } & \multirow[b]{2}{*}{ Measure ${ }^{a}$} & \multicolumn{3}{|c|}{ Signal-to-Noise Ratio } \\
\hline & & & 20 & 10 & 5 \\
\hline \multirow[t]{2}{*}{$\begin{array}{l}B \\
\text { (ms) }\end{array}$} & Curve Fit & $\begin{array}{l}\epsilon_{\text {abs }} \\
\epsilon\end{array}$ & $\begin{array}{r}0.3 \\
-0.1\end{array}$ & $\begin{array}{l}0.6 \\
0.0\end{array}$ & $\begin{array}{l}1.3 \\
0.2\end{array}$ \\
\hline & Simple & $\begin{array}{l}\epsilon_{\text {abs }} \\
\epsilon\end{array}$ & $\begin{array}{r}8.6 \\
-7.9\end{array}$ & $\begin{array}{r}13.0 \\
-11.6\end{array}$ & $\begin{array}{r}25.0 \\
-23.0\end{array}$ \\
\hline \multirow[t]{2}{*}{$\begin{array}{l}X \min \\
(\mathrm{ms})\end{array}$} & Curve Fit & $\begin{array}{l}\epsilon_{\text {abs }} \\
\epsilon\end{array}$ & $\begin{array}{l}1.1 \\
0.8\end{array}$ & $\begin{array}{l}1.6 \\
1.3\end{array}$ & $\begin{array}{l}1.9 \\
1.4\end{array}$ \\
\hline & Simple & $\begin{array}{l}\epsilon_{\mathrm{abs}} \\
\epsilon\end{array}$ & $\begin{array}{l}2.0 \\
1.0\end{array}$ & $\begin{array}{l}2.4 \\
1.3\end{array}$ & $\begin{array}{l}4.5 \\
0.9\end{array}$ \\
\hline
\end{tabular}

${ }^{a} \epsilon$ is mean error, $\epsilon_{a b s}$ is mean absolute error.

downward until the first local minimum was found, resulting in a systematic underestimation of LVET. Conversely, programming that would have carried out the search starting from left of the B point would have resulted in the detection of the position of the event left of the actual B point, thus systematically overestimating LVET and leading to an inflation of stroke volume. Clearly, the curve fitting method enables accurate point detection in the face of a wide SNR range. Due to the excellent visibility and symmetry of the $\mathrm{X}$ wave in the template signal, the difference in mean error between the curve fitting and local minima technique is not very large in detecting the $\mathrm{X}$ wave minimum. Nevertheless, the curve fitting technique still exhibits less tendency than simple methods to be influenced by superposition of noise.

Although the mean error $d Z / d t$ peak localization displays only relatively small differences between simple maximum detection and matched filtering, the performance

TABLE 2. Precision of simple and matched filtering methods: Effect of noise on the position and amplitude of the $d Z / d t$ max.

\begin{tabular}{|c|c|c|c|c|c|}
\hline \multirow[b]{2}{*}{ Event } & \multirow[b]{2}{*}{ Method } & \multirow[b]{2}{*}{ Measure $^{a}$} & \multicolumn{3}{|c|}{ Signal-to-Noise Ratio } \\
\hline & & & 20 & 10 & 5 \\
\hline \multirow{4}{*}{$\begin{array}{l}d Z / d t \text { max position } \\
\text { (ms) }\end{array}$} & Matched Filt. & $\epsilon_{\mathrm{abs}}$ & 0.0 & 0.1 & 0.3 \\
\hline & & $\epsilon$ & 0.0 & -0.1 & -0.1 \\
\hline & Simple & $\epsilon_{\mathrm{abs}}$ & 2.8 & 3.4 & 4.1 \\
\hline & & $\epsilon$ & 0.1 & 0.9 & 1.3 \\
\hline \multirow{4}{*}{$\begin{array}{l}d Z / d t \text { max amplitude } \\
(\mathrm{m} \Omega / \mathrm{s})\end{array}$} & Matched Filt. & $\epsilon_{\mathrm{abs}}$ & 10.7 & 23.4 & 51.5 \\
\hline & & $\epsilon$ & -4.1 & -0.7 & 5.4 \\
\hline & Simple & $\epsilon_{\mathrm{abs}}$ & 74.0 & 140.3 & 428.0 \\
\hline & & $\epsilon$ & 52.6 & 82.9 & 328.4 \\
\hline
\end{tabular}

${ }^{a} \epsilon$ is mean error, $\epsilon_{a b s}$ is mean absolute error. 
of simple maximum detection shows a clear tendency to be affected by increasing noise levels (see Table 2). Major differences in performance can be seen for the determination of $d Z / d t_{\max }$. The $d Z / d t$ maximum of the analyzed signals was in the range of about $1300 \mathrm{~m} \Omega / \mathrm{s}$. As can be seen in Table 2, the absolute error for matched filter detection stays below $5 \%$ even for the worst signal-to-noise ratio of 5. Conversely, for the simple technique, the absolute error for SNR $=5$ is larger than $30 \%$.

These test results reveal a substantial improvement in precision of the new signal processing techniques over the commonly used techniques. With errors for the determination of $d Z / d t_{\max }$ in the range of up to $30 \%$, previous difficulties related to the reliability of impedance cardiography may to a large extent be attributed to signal processing.

\section{RESULTS}

Based on the newly developed signal processing techniques, an entirely computerized detection system was developed and tested in a laboratory study both against previous techniques and against the performance of a human operator, who was considered as the standard for event detection. Unfortunately, invasive studies using electromagnetic flow probes could not be performed on human subjects, and none of the other available invasive techniques such as thermodilution offers beat-to-beat measurement with acceptable precision. The selected standard, the human operator, offers excellent reliability and precision, but only if the signals show good quality with minimal noise, artifact, and signal distortion. An additional limitation is that the human operator is not able to perform the important task of eliminating respiratory modulation from the signals.

Determination of systolic time intervals is best suited to test the precision of the new techniques, since these can relatively easily be determined by a human operator. Pre-ejection period (PEP), left ventricular ejection time (LVET), and electromechanical systole $\left(Q S_{2}\right)$ have been used to provide an estimate of the performance of the system on real signals. Included in the analysis was an investigation of how different definitions of cardiac events influence the outcome of the parameters. The beginning of the electrical ventricular systole, for example, is indicated in the ECG by the onset of the $Q$ wave (see Fig. 1). Probably due to the difficulty in determining slow wave onsets, some investigators have instead chosen to use the minimum of the $Q$ wave located at the foot of the $R$ wave $(13,19,21)$, leading to a systematic underestimation of PEP. Our analysis, based on the measurement on ten healthy male and female individuals free of cardiopathologic history, with a mean age of 37 years, showed a mean systematic error of $15.2 \mathrm{~ms}$ between $\mathrm{Q}$ wave onset and minimum. For the normal range of PEP values (80-120 ms), this represents a difference of well above $10 \%$. The mean difference between our computerized detection of $Q$ wave onset and the visual detection by an expert operator was $0.3 \mathrm{~ms}$.

The end of PEP and beginning of LVET has to be determined by the detection of the onset of blood ejection from the left ventricle. Commonly, the B point of the impedance signal is considered to mark this event; zero-crossing of $d Z / d t$ prior to the sharp upstroke has, however, also been considered as a possible event location. Again, the two definitions lead to systematic differences in the cardiac time interval, in this case affecting two parameters, PEP and LVET. This has a dramatic influence on the ratio of PEP/LVET, which has been found to be more useful as an index of 
left ventricular contractility than either of these time intervals alone (11). Incorrect determination of the transition between PEP and LVET influences numerator and denominator antithetically, thus amplifying the error. Tests of our computer event detection of the $\mathrm{B}$ point showed high precision: mean difference between operator and computer detection was $1.5 \mathrm{~ms}$. Comparing the $\mathrm{B}$ point and zero-crossing detection of the aortic opening showed that zero-crossing leads to greater variance of the parameters. It also tends to underestimate PEP and to overestimate LVET by $10 \mathrm{~ms}$ on average with the consequence of lower precision, inflated stroke volume and an approximately $10 \%$ reduction of contractility index for the normal range of cardiac time intervals.

The endpoint of LVET is commonly attributed to the $X$ wave of the impedance cardiogram. Since the $\mathrm{X}$ wave is, however, a rather extended event, further specification is necessary. So far, the minimum of the $X$ wave has been used as the event signalling the end of the ejection period. Other possible markers are the onset of the $\mathrm{X}$ wave, and the onset of the maximal vibration of the second heart sound in the phonocardiogram (PCG). The presented study recorded ECG, PCG, and ICG in the above-mentioned test group to examine on a beat-to-beat basis the effect of using the minimum of the $X$ wave to denote the end of LVET. It also examined the temporal relationship of the minimum of the $X$ wave to the onset of the second heart sound and also to the onset of the $\mathrm{X}$ wave.

Signals were recorded for a one minute interval at the end of each of the following three periods: 20 minute rest period, 3 minute period of preparation of a speech (speech preparation) on a threatening topic, and a three minute period during which the speech was delivered. In previous studies this speech task has been shown to elicit large increases in blood pressure and heart rate (16). Accordingly, in this study during speech preparation and talk, respectively, subjects displayed mean systolic blood pressure increase of $17 \mathrm{mmHg}$ and $32 \mathrm{mmHg}$ which was accompanied by a diastolic pressure increase of 6 and $16 \mathrm{mmHg}$ relative to resting levels. Heart rate increased 9 and $12 \mathrm{bpm}$ during speech preparation and talk, respectively.

Table 3 compares the onset of the second heart sound with the $X$ wave onset and $\mathrm{X}$ wave minimum as reference points for aortic valve closure. A large difference between the S2 and X minimum can be seen of approximately $23 \mathrm{~ms}$, with the S2 detected earlier than the $\mathrm{X}$ minimum. Alternatively, the $\mathrm{X}$ onset corresponds very closely in time with S2 onset occurring within about $4 \mathrm{~ms}$ of the S2 onset. The impact of the use of the $X$ minimum on LVET is to overestimate this interval by about $8-10 \%$. Therefore, the contractility index, PEP/LVET, may be underestimated by

TABLE 3. Comparison of the Difference in ms of the Positions of $\$ 2, X$ onset and $X$ minimum.

\begin{tabular}{llccc}
\hline \multicolumn{1}{c}{ Event } & Measure $^{\mathrm{a}}$ & Rest & Speech Prep & Speech Talk \\
\hline S2-Xon & $\epsilon$ & 4.4 & 3.8 & 5.0 \\
S2-Xmin & $\epsilon$ & -22.7 & -24.5 & -21.2 \\
Xon-Xmin & $\epsilon$ & -30.2 & -31.7 & -33.5 \\
LVET $_{\text {B-Xon }}$ & $\mathrm{M} \pm \mathrm{se}$ & $260.5 \pm 3$ & $258.5 \pm 4$ & $255.5 \pm 6$ \\
LVET $_{\mathrm{B}-\mathrm{Xmin}}$ & $\mathrm{M} \pm \mathrm{se}$ & $290.7 \pm 4$ & $290.3 \pm 4$ & $289.0 \pm 6$ \\
\hline
\end{tabular}

${ }^{a} \epsilon$ is mean error, $M \pm s e$ is mean \pm standard error. 
TABLE 4. Comparison of stroke volume in $\mathrm{ml}$ using left ventricular ejection times (LVET) measured from $B$ point to $X$ onset or from $B$ point to $X$ minimum.

\begin{tabular}{ccccc}
\hline Event & Measure $^{a}$ & Rest & Speech Prep & Speech Talk \\
\hline SV $_{\text {Xon }}-$ SV $_{\text {Xmin }}$ & $\epsilon$ & -7.9 & -8.5 & -9.3 \\
SV $_{\text {(LVET B-Xon) }}$ & $\mathrm{M} \pm \mathrm{se}$ & $69.8 \pm 2$ & $70.5 \pm 3$ & $68.9 \pm 6$ \\
SV $_{\text {(LVET B-Xmin) }}$ & $\mathrm{M} \pm \mathrm{se}$ & $77.7 \pm 2$ & $79.0 \pm 3$ & $78.2 \pm 6$ \\
\hline
\end{tabular}

${ }^{a} \epsilon$ is mean error, $M \pm s e$ is mean \pm standard error.

about $25 \%$ due to the accumulated error of underestimating PEP when using Q minimum instead of Q onset and overestimating LVET when using $X$ minimum instead of $\mathrm{X}$ onset. The difference between $\mathrm{X}$ onset and minimum cannot be trivialized as can be seen in Table 4. When stroke volume was calculated with LVET measurement from the B point to the $\mathrm{X}$ minimum (see Table 4), a difference of about 8-9 $\mathrm{ml}$ was observed relative to the stroke volume derived using the LVET as measured from the $B$ point to the $\mathrm{X}$ onset.

The present results suggest that studies which report that the Kubicek equation overestimates stroke volume may have been exposed to systematic error in signal event definition resulting in a $10 \%$ overestimation of stroke volume $(7,12,17)$. The data in this study suggest that greater reliability and precision of cardiovascular parameters may be achieved from a system using the new signal processing techniques proposed in this paper together with the correct definition of cardiac time intervals.

\section{MODELS}

The major conclusion of our investigations are that the proposed signal processing techniques increase reliability and precision of event recognition and amplitude measurement, but that the determination of stroke volume in cases of unusual shape of the impedance cardiogram still needs some improvements. The reason for shifts of the B point toward or away from the $d Z / d t$ peak (and its significance for stroke volume determination) and double peaks in the impedance signal need further clarification. In order to address some remaining unanswered questions, including the validity of impedance cardiography, we have compared the impedance signal with invasive electromagnetic flow measurement in rabbits. Independent from the issue of the extent to which rabbit and human cardiovascular systems show comparable results, basic assumptions of the model for stroke volume determination can certainly be verified. Flow and impedance signal shown in Fig. 10 indicate that the signal shapes correspond very well to those found in humans (see Fig. 11).

Previous noninvasive studies suggested that the observed impedance changes result almost completely from aortic blood flow (10). According to this hypothesis, interruption of the aortic blood flow should also lead to zero impedance signal. Figure 12 shows synchronous flow, ECG, and impedance tracings with interrupted blood flow. The marker indicates the time where the aorta, after being clamped to interrupt flow, has been opened again leading to the subsequent restoration of flow. It can be clearly seen that the impedance signal shows reduced amplitude for the zero flow interval, but does not disappear completely. Thus, the measurement suggests 


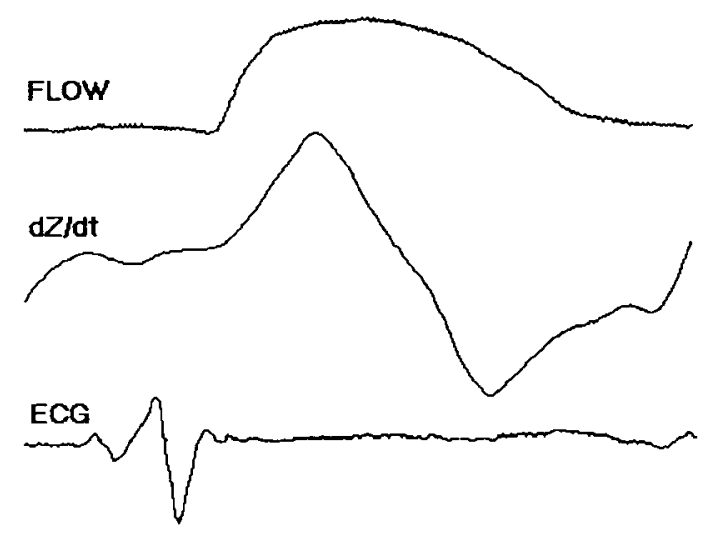

FIGURE 10. Electromagnetic measurement of aortic flow in a rabbit. The impedance cardiogram shows similar shape as the human ICG in Fig. 11.

that the impedance signal does not result exclusively from aortic blood flow. As pulmonary flow will also be interrupted after aortic clamping, the remaining signal cannot be attributed to the pulmonary flow. A possible interpretation of this situation is that the contraction of the heart and the associated change of thoracic geometry also contribute to the impedance signal. This would be in accordance with previous observations of impedance changes during the isovolumetric contraction phase.

\section{CONCLUSION}

The new signal processing techniques presented in this paper substantially improve the precision of event detection on the impedance cardiogram in the face of varying levels of noise and artifact. When systematic errors are avoided by detecting $Q$ wave onset, $\mathrm{B}$ point and $\mathrm{X}$ wave onset, cardiac parameters such as stroke volume and sys-

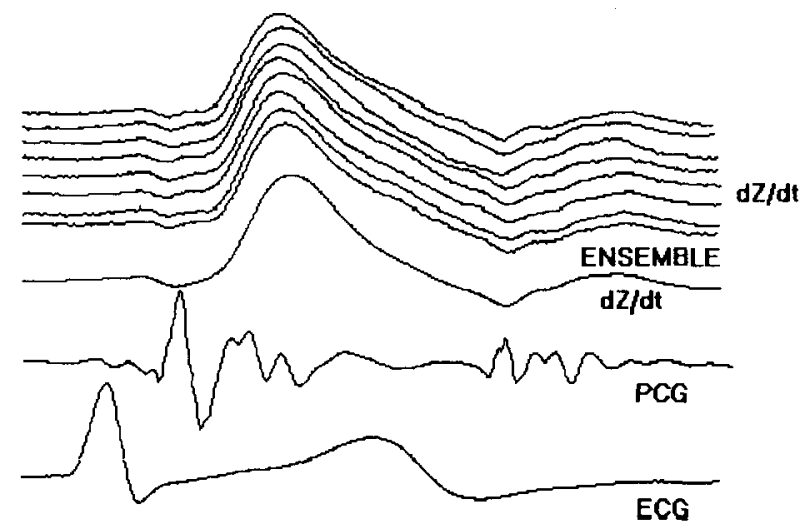

FIGURE 11. Improvement of SNR by coherent ensemble averaging of the impedance cardiogram of a human subject. PCG and ECG are shown for reference. 


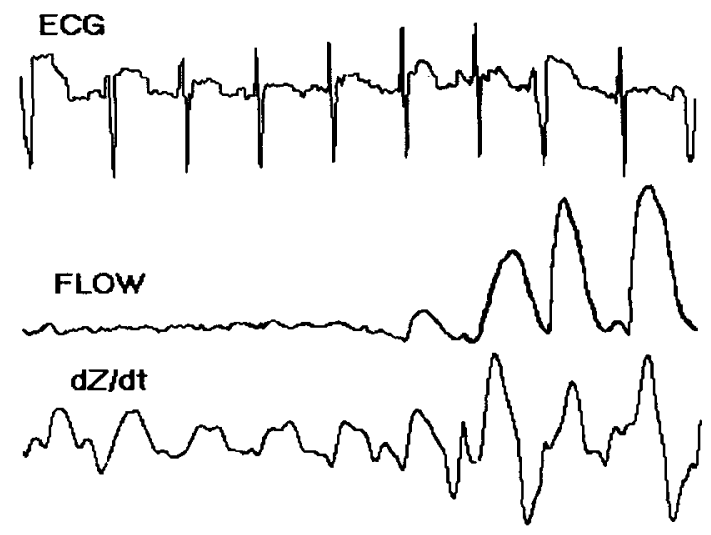

FIGURE 12. Electromagnetic flow measurement in a rabbit. Initially, the aorta was clamped, the flow ceased but the $d Z / d t$ signal was not completely diminished: upon opening the aorta again, flow was restored, the $d Z / d t$ signal recovers to normal amplitude and shape.

tolic time intervals derived from the impedance cardiogram will more reliably reflect actual values. Although comparison of impedance derived values using the techniques described above with invasive electromagnetic flow measurements has yet to be performed, the presented results show that the new signal processing techniques provide the tools for future assessment of impedance cardiography as an instrument of medical diagnostics and intensive care monitoring.

\section{REFERENCES}

1. Appel, P.L.; Kram, H.B.; MacKabee, J.; Flemming, A.W.; Shoemaker, W.C. Comparison of measurements of cardiac output by bioimpedance and thermodilution in severely ill surgical patients. Crit. Care Med. 14:933-935; 1986.

2. Aust, P.E.; Belz, G.G.; et al. Comparison of impedance cardiography and echocardiography for measurement of stroke volume. Eur. J. Clin. Pharmacol. 23:475; 1982.

3. Bernstein, D.P. Continuous non-invasive real-time monitoring of stroke volume and cardiac output by thoracic electrical bioimpedance. Crit. Care Med. 14:10:898-901; 1986.

4. Bleicher, W.; Steil, E.; Fiderer, F.; Wolf, M.; Faust, U. Non-invasive monitoring of heart function with the aid of the automatically processed impedance cardiogram. Froceedings of the Vth ICEBI. Tokyo, 57-59; 1981.

5. Denniston, J.C.; Maher, J.T.; Reeves, J.T.; et al. Measurement of cardiac output by electrical impedance at rest and during exercise. J. Appl. Physiol. 40:91; 1976.

6. Donovan, K.D.; Dobb, G.L.; Woods, W.P.D.; et al. Transthoracic electrical impedance and thermodilution methods for measuring cardiac output. Crit. Care Med. 14:1038-1044; 1986.

7. Edmunds, A.T.; Godfrey, S.; Tooley, M. Cardiac output measured by transthoracic impedance cardiography at rest, during exercise and at various lung volumes. Clin. Sci. 63:107; 1982.

8. Keim, J.H.; Wallace, J.M.; Thurston, H.; et al. Impedance cardiography for determination of stroke index. J. Appl. Physiol. 41:797; 1976.

9. Kubicek, W.G.; Karnegis, J.N.; Patterson, R.P.; Witsoe, D.A.; Mattson, R.H. Development and evaluation of an impedance cardiac output system. Aerospace Medicine 39:248-252; 1966.

10. Lamberts, R.; Visser, K.R.; Zijlstra, W.G. Impedance Cardiography. Assen, The Netherlands: Van Gorcum; 1984, pp. 94-106.

11. Larsen, P.B.; Schneiderman, N.; Pasin, R.D. Physiological bases of cardiovascular psychophysiology. In: Coles, M.; Danchin, E.; Porges, S., eds. Psychophysiology: Systems, Processes and Applications. New York: Guilford Press; 1986, pp. 122-165. 
12. Miller, J.C.; Horvath, S.M. Impedance cardiography. Psychophysiology. 40:80; 1978.

13. Muzi, M.; Jeutter, D.C.; Smith, J.J. Computer-automated impedance-driven cardiac indexes. IEEE Transactions on Biomedical Engineering. BME-33, No. 1:42-47; 1986.

14. Nagel, J. Method and apparatus for identifying recurring signal patterns. US patent No. 4,211,237; 1980.

15. Ritz, R.; Furrer, M. Trendbeobachtung des schlagvolumens bei intensivpatienten aufgrund der kontinuierlich registrierten elektrischen impedanz. Intensivmed. 21:132; 1984.

16. Saab, P.G.; Matthews, K.A.; Stoney, C.M.; McDonald, R.H. Premenopausal and postmenopausal women differ in their cardiovascular and neuroendocrine responses to behavioral stressors. Psychophysiology, in press.

17. Secher, N.J.; Thomsen, A.; Arnsbo, P. Measurement of rapid changes in cardiac stroke volume. An evaluation of the impedance cardiography method. Acta. Anaesth. Scand. 21:353; 1977.

18. Sheps, D.S.; Petrovick, M.L.; Kizakevich, P.N.; Wolfe, C.; Craige, E. Continuous noninvasive monitoring of left ventricular function during exercise by thoracic impedance cardiography-automated derivation of systolic time intervals. American Heart Journal. 103:519-524; 1982.

19. Sherwod, A.; Allen, M.T.; Langer, A.W. Evaluation of beta-adrenergic influences on cardiovascular and metabolic adjustments to physical and psychological stress. Psychophysiology. 23:89-104; 1986.

20. Sherwood, A.; Allen, M.T.; Fahrenberg, J.; Kelsey, R.M.; Lovallo, W.R.; van Doornen, L.J.P. Methodological guidelines for impedance cardiography. Psychophysiology, in press.

21. Sramek, B.B. Cardiac output by electrical bioimpedance. Medical Electronics. April:93; 1982.

22. Zhang, Y.; Qu, M.; Webster, J.G.; Tompkins, W.J.; Ward, B.A.; Bassett, D.R. Cardiac output monitoring by impedance cardiography during treadmill exercise. IEEE Transactions on Biomedical Engineering, BME33:1037-1042; 1986.

\section{NOMENCLATURE}

PEP = Pre-ejection period

LVET $=$ Left ventricular ejection time

$\mathrm{ECG}=$ Electrocardiogram

ICG = Impedance cardiogram

PCG = Phonocardiogram

$\rho_{b} \quad=$ Resistivity of blood

$\mathrm{SV}=$ Stroke volume

$L \quad=$ Distance between measuring electrodes

$Z_{o} \quad=$ baseline impedance between recording electrodes

$d Z / d t=$ First time derivative of thoracic impedance

$Z \quad=$ Thoracic impedance

$B=$ Point in the ICG indicating onset of left ventricular ejection time

$Q S_{2} \quad=$ Total electromechanical systole

$S 1 \quad=$ First heart sound

$S 2 \quad=$ Second heart sound 